\title{
BIO Inspired Clustering and Routing for Wireless Sensor Networks
}

\author{
Ruby Chauhan \\ Research Scholar, \\ Punjab Technical University, \\ DAVIET, Jalandhar (India)
}

\author{
Rajeev Kumar \\ Asst. Professor (IT), \\ Punjab Technical University, \\ DAVIET, Jalandhar (India)
}

\begin{abstract}
A quality literature survey was carried out in the area of Wireless Sensor Network and it was observed that leach protocol which has been proposed in the literature phase continued some problem, i.e. cluster construction and cluster head selection. PEGASIS overcome shortcomings of LEACH but PEGASIS still lack in outperforms the other two protocols in terms of system lifetime and quality of network on large scale wireless sensor network. Using BFO on LEACH, as each cluster head directly transmit data to base station the network consumes more energy that decreases the network life time and also affect the scalability of nodes in Network as well as Deployment of nodes in the network field.. In this paper we proposed a new protocol to improve the existing PEGASIS protocol for clustering and routing using Bacterial Foraging Optimization simultaneously to evaluate and compare the proposed approach with existing protocol based on parameters like Network lifetime, Energy Consumption, Throughput, Number of alive nodes. In the end we conclude this paper with future research and conclusion that there is need to expanded further this procedure, to apply the quantum acted PSO with BFO for selecting the suitable group head in the remote sensor systems
\end{abstract}

\section{General Terms}

Clustering, Algorithms, Routing, chemo taxis etc.

\section{Keywords}

First Node Dead, Last Node Alive, Swims and Tumble

\section{INTRODUCTION}

Wireless sensor network (WSN) have numerous sensor nodes capacitive to sense, compute and communicate. Sensors consumes energy to gather data, sending and receiving data among various nodes within a network. Data communication between the senor nodes takes maximum energy consumption in WSN. So, it is necessary to design WSN with intelligence. Deployment of sensor nodes held uniformly with accordance to some specific plan to achieve such application-specific task. WSN is different from other established wireless networks like mobile ad hoc networks (MANET) and cellular systems. WSNs have vast field of new challenges in the development and application due to its exclusive features and storage limitations.

Clustering: Clustering process have been applied with hierarchical structures to sensor networks for getting the network performance by reduced energy consumption. Basic methodology of clustering is to collect nodes around a cluster head for connectivity with all other cluster members. Clustering means deploying nodes randomly over the network joins together to accomplish the task. In clustering the $\mathrm{CH}$ broadcast the signal to all nodes, on receiving the signals from different $\mathrm{CHs}$, nodes reply to $\mathrm{CHs}$ with their respective nodeid with more signal strength so a cluster is formed.

\section{LITERATURE SURVEY}

In PEGASIS, every hub speak just with a nearby neighbor and alternates transmitting to the base station which decreases the measure of vitality spent in each round. Accordingly, PEGASIS performs 100 to $300 \%$ superior to anything LEACH when $1 \% 20 \%$ half and $100 \%$ of hubs kick the bucket for distinctive sizes and topologies.[1]Another kind of steering convention for Wireless Sensor Network called PECRP (Power-proficient Clustering Routing Protocol), which is suitable to long-separation and complex information transmission and for settled sensor hubs of WSN. PECRP utilizes multi-bounce transmission for information transmission, that is called "circle domino effect in light of separation to BS (Base Station)" to adjust the vitality utilization in hubs. [2]A clock is acquainted with verify choosing the ideal sensor hub as bunch head in procedure of setting up cluster. During information transmission, utilizing single jump and multi-bounce half breed steering to convey can use vitality all the more viably and equally. [3]Distance Aware Intelligent Clustering (DAIC). is plunging the system in two tiers, Primary level and optional level and selecting the high vitality $\mathrm{CHs}$ at the most brief separation from the BS. In it BS finds its vertical separation from every hub, the vertical separation recognizes the $\mathrm{CHs}$ and Gateways $\mathrm{CHs}$ in the levels. [4]A PSO-based convention is utilized to locate the ideal number of bunch heads to augment the system vitality proficiency and the same time, the system scope and connections quality are taken into record .Broad re-enactment demonstrate that the proposed convention beats the surely understood bunch based sensor system conventions as far as normal vitality utilization while in the meantime has adequate information bundles throughput. The sensor hubs were conveyed haphazardly in a zone of to minimize the quantity of CHs.[5]An energy independent cluster routing (EICR) is advanced to defeat the disadvantages of LEACH. Numerical reproduction results demonstrate that the got methodology can empower node with the same remaining energy, adjust the node's energy, and prolongs the system lifetime. The changes of EICR incorporate two angles: (1) guarantee that the hub with greatest remaining energy gets to be $\mathrm{CH}$; (2) guarantee that the $\mathrm{CH}$ in every round is with the normal head number. The dispersion of cluster is more uniform. [6]An enhanced energy productive PEGASIS-based convention (IEEPB) is proposed in this paper. IEEPB embraces new technique to fabricate chain, and uses weighting system when selecting the pioneer node. In this paper, proposed an energy effective Cluster head determination calculation for remote sensor systems. By utilizing a Ridge system to choose best clusters head, RMCHS dependably picks $\mathrm{CHs}$ out from nodes with higher leftover energy.[7] a super cluster head $(\mathrm{SCH})$ is chosen among the $\mathrm{CHs}$ who can just send the data to the 
versatile BS by picking suitable fuzzy descriptors. Fuzzy surmising motor (Mamdani's tenet) is utilized to choose the opportunity to be the SCH. The outcomes have been gotten from NS-2 test system and demonstrates that the proposed convention performs superior to anything LEACH convention as far as First nodes bites the dust, Half node alive, better strength and better lifetime. [8]

\section{PROPOSED DEPLOYMENT METHOD}

1. Initialize number of nodes, energy, and location of base station for the network.

2. Construct the clusters using LEACH mechanism.

3. Select the cluster head using bacterial foraging optimization i.e. finding the cluster head that have highest fitness value among all other nodes in the cluster.

4. Find the Farthest cluster head from the base station using bubble sort method and start constructing chain from that cluster head using BFO.

5. The least distant cluster head from the base station will be the leader among all other cluster heads.

6. The leader cluster head will transmit the data to the base station using BFO.

\section{ABOUT BACTERIAL FORAGING OPTIMIZATION (BFO)}

$\mathrm{BFO}$ is inspired by the social foraging behavior of Escherichia coli. (Rod shaped bacteria from the family of entero bacteriaceae).

As shown in fig 1.1 Escherichia coli bacteria (E. Coli is a single celled organism that is in our gut or small intestine of human body. These cells are $45 \mathrm{~nm}$ in diameter and equipped with a set of rotary motor. Each motor drives a thin long filament. This filament is named as Flagella help E. coli bacteria to tumble and swim. E.Coli bacterial finds the nutrient rich location in human intestine. An E. Coli bacterium has two types of movements: swimming and tumbling. In swimming movement, the bacteria moves in a straight line in a given direction, while in tumbling the bacteria randomly changes its direction of movement. BFA has three steps: a) Elimination step, b) Reproduction step and c) Chemo taxis step. The bacteria initially start with any random position and evaluate the objective function to determine the movement in which direction. Then it moves in a particular direction again and evaluates the objective function. If it is better than the last one it continuously moves in the same direction until it gets the bad objective function. This whole step is Chemotaxis step. After a specific number of Chemotaxis steps, the reproduction of the bacteria takes place in which the two bacteria combines to form a healthy bacteria and next is the elimination-dispersal step in which the bacteria splits into two bacteria and the non-healthy bacteria is eliminated in this step. These steps are continuously repeated up to specific number of times until the objective function is reached.

BFO has been widely accepted global optimization algorithms of current interest for distributed optimization.

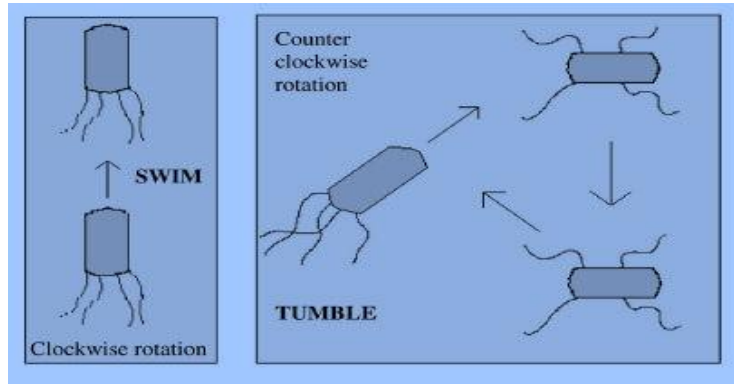

Figure 1.1 Bacterium swims and tumble. Reference paper[13]

When flagella are rotated in clockwise direction the bacterium swims very fast in forward direction. Where flagella rotate in counter clockwise direction bacterium starts tumble in search of nutrient in new location. A BFO algorithm follows three Principles. Chemo taxis, Reproduction, Elimination/dispersal. Chemo taxis: The process in which bacteria moves and search for nutrient in order to maximize the energy level is called chemo taxis. Swimming and tumbling are the two modes of movement for the life time of a bacterium to

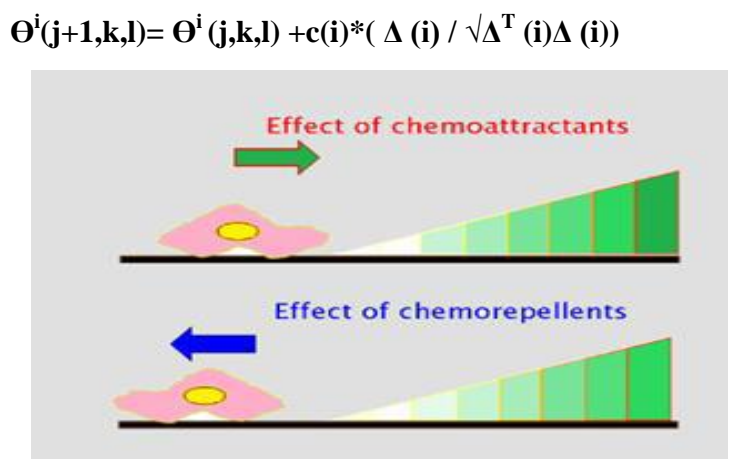

Figure 1.2 Effects of chemo taxis- Reference paper[13]

Above equation represent the chemotactic movement of the bacteria, where $\theta \mathrm{i}(\mathrm{j}, \mathrm{k}, \mathrm{l})$ is represented by ith bacterium at jth chemotactic, kth reproduction and lth elimination-dispersal steps. C (i) is the size of the steps taken in random direction and indicates a vector in the arbitrary direction whose element lies between $[-1,1]$.

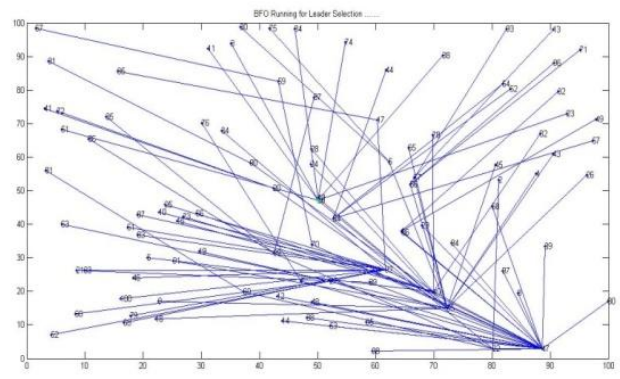

Figure 1.3 Clustering and Leader selection by BFO

Above figure shows Clustering pattern using BFO and Leader selection by BFO, Node pointing with green dot is selected as leader for a particular round. 


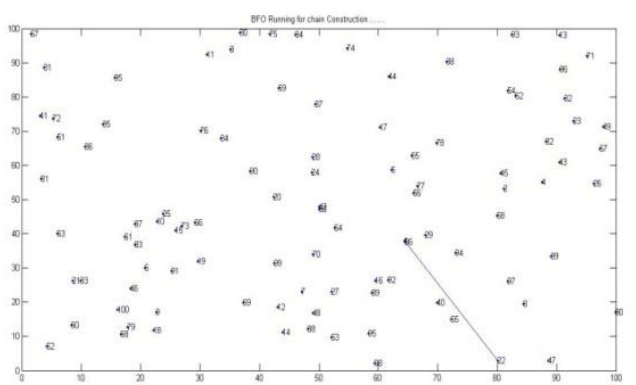

Figure 1.4 Chain construction with BFO

This graph shows chain construction with BFO methodology which is quite different from traditional chain construction by Pegasis and it is also shown that in Pegasis with BFO.

\section{PEGASIS ALGORITHM}

\subsection{Initialize}

- Define the Initial and Destination Node called $\mathbf{S i}$ and Di

- Find the MaxDistance and MinDistance Path between $\mathbf{S i}$ and $\mathbf{D i}$

- $\quad$ Start Communication from Node $\mathbf{S i}$

- $\quad$ Find All N Neighbor Nodes of $\mathbf{S i}$ called Neighbor $\mathbf{i}<$ DistanceVector

\subsection{Main processing}

- For $\mathrm{I}=1$ to $\mathrm{N}$

- Find MinDistance, MinEnergy, Cidel for all the Neighbors nodes

- Ithe parameter for making the decision in which route data will be transfer that called Cidel. Cidel is actually as the response ofthenode, means how quickly node is giving response

- $\quad$ CIdel $=$ Throughput - Ceffective

- It is define as expected and actual response of the node. Ceffective is defined as distance $^{*}$ Transmission

distance $*$ Transmission*overhead . Where overhead value is supposed to be .02.Therefore. Throughput is defined as throughput $=$ (distance +Congestion*distance) *Transmission distance $*$ Transmission*overhead $* m$ when its value is less then Congestion<.5 then $m$ will be 2othervise $m$ will be $3 / /$

- If (Energy (Neighbour)) =MinEnergy\&\&Cidel (Neighbour)) <= Threshold \&\& Distance(Neighbour) $=$ MinDistance

- $\quad$ Set Neighbour node as Next node

- Else if (Energy(Neighbour))=MinEnergy and Distance $($ Neighbour $)=$ MinDistance

- $\quad$ Set Neighbour node as Next node

- $\quad$ Else if Distance(Neighbour $)=$ MinDistance

- Set Neighbour node as Next node

- $\quad$ Else $($ Energy $($ Neighbour $))=$ MinEnergy

- $\quad$ Set Neighbour node as Next node

\subsection{Finalize}

Set $\mathbf{S i}=$ Selected Neighbour and repeat process for $\mathbf{n}$ rounds

\subsection{Parameters}

[Step 1] Initialize parameters $p, S, N_{c}, N_{S}, N_{r e}, N_{e d}, P_{e d}$, $C(i)(i=1,2 \ldots S), \theta^{i}$

\subsection{Algorithm}

[Step 2] Elimination-dispersal loop: $l=l+1$

[Step 3] Reproduction loop: $k=k+1$

[Step 4] Chemotaxis loop: $j=j+1$

[a] For $i=1,2 \ldots \mathrm{S}$ take a chemotactic step for bacterium $i$ as follows.

[b] Compute fitness function, $J(i, j, k, l)$.

Let, $J(i, j, k, l)=J(i, j, k, l)+J_{C C}\left(\theta^{i} .(j, k, l), P(j, k, l)\right) i$ (i.e. add on the cell-to cell

attractant-repellant profile to simulate the swarming behavior)where, $J_{C C}$ is defined in (2).

[c] Let $J_{\text {last }}=J(i, j, k, l)$ to save this value since we may find a better cost via a run.

[d] Tumble: generate a random vector $\mathrm{D}(i) \hat{\mathrm{I}} \hat{\mathrm{A}} p$ with each element $\operatorname{D} m(i), m=1,2, \ldots, p$, a random number on $[-1,1]$

[e] Move: Let

$\theta^{i}(j+1, k, l)=\theta^{i}(j, k, l)+C(i) \frac{\Delta(i)}{\sqrt{\Delta^{T}(i) \Delta(i)}}$

This results in a step of size $C(i)$ in the direction of the tumble for bacterium $i$

[f] Compute $J(i, j+1, k, l)$ and let

$J(i, j+1, k, l)=J(i, j, k, l)+J_{C C}\left(\theta^{i}((j+1, k, l), P(j+1, k, l))\right.$

[g] Swim

i) Let $m=0$ (counter for swim length)

ii) While $m<s N$ (if have not climbed down too long).

- $\quad$ Let $m=m+1$.

- If $\mathbf{J}(i, j+1, k, l)<J_{\text {last }}$ (if doing better), let $J_{\text {last }}(i$, $j+1, k, l)$ and let

$\theta^{i}(j+l, k, l)=\theta^{i}(j, k, l)+C(i) \frac{\Delta(i)}{\sqrt{\Delta^{T}(i) \Delta(i)}}$

And use this $\theta^{i}((j+1, j, k, l)$ to compute the new $\mathbf{J}(i, j+1, k$, $l)$ as we did in $[\mathrm{f}]$

- Else, let $\mathrm{m}=\mathrm{N}_{\mathrm{s}}$. This is the end of the while statement.

[h] Go to next bacterium $(i+1)$ if $i \neq S$ (i.e., go to [b] to process the next bacterium)

[Step 5] If $j<N_{c}$, go to step 4. In this case continue chemotaxis since the life of the bacteria is not over.

[Step 6] Reproduction:

[a] For the given $k$ and $l$, and for each $i=1,2, \ldots, S$, let 


$$
J_{\text {healt } h}^{i}=\sum_{j=1}^{N_{c+1}} J(i, j, k, l)
$$

be the health of the bacterium $i$ (a measure of how many nutrients it got over its lifetime and how successful it was at avoiding noxious substances). Sort bacteria and chemotactic parameters $C(i)$ in order of ascending cost Jhealth (higher cost means lower health).

[b] The $S_{r}$ bacteria with the highest $J$ health values die and the remaining $S_{r}$ bacteria with the best values split (this process is performed by the copies that are made are placed at the same location as their parent).

[Step 7] If $k<N_{r e}$, go to step 3. In this case, we have not reached the number of specified reproduction steps, so we start the next generation of the chemotactic loop.

[Step 8] Elimination-dispersal: For $i=1,2 \ldots, S$ with probability $P_{e d}$, eliminate and disperse eachbacterium (this keeps the number of bacteria in the population constant). To do this, if a bacterium is eliminated, simply disperse another one to a random location on the optimization domain. If $l<N_{e d}$, then go to step 2; otherwise end.

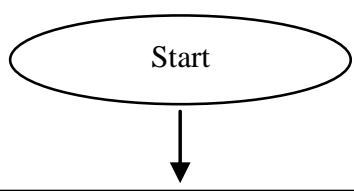

Initialize number of nodes, energy and location to the base station

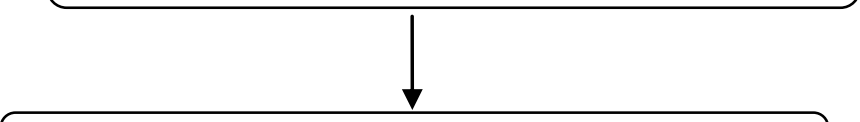

Construct the clusters using LEACH mechanism

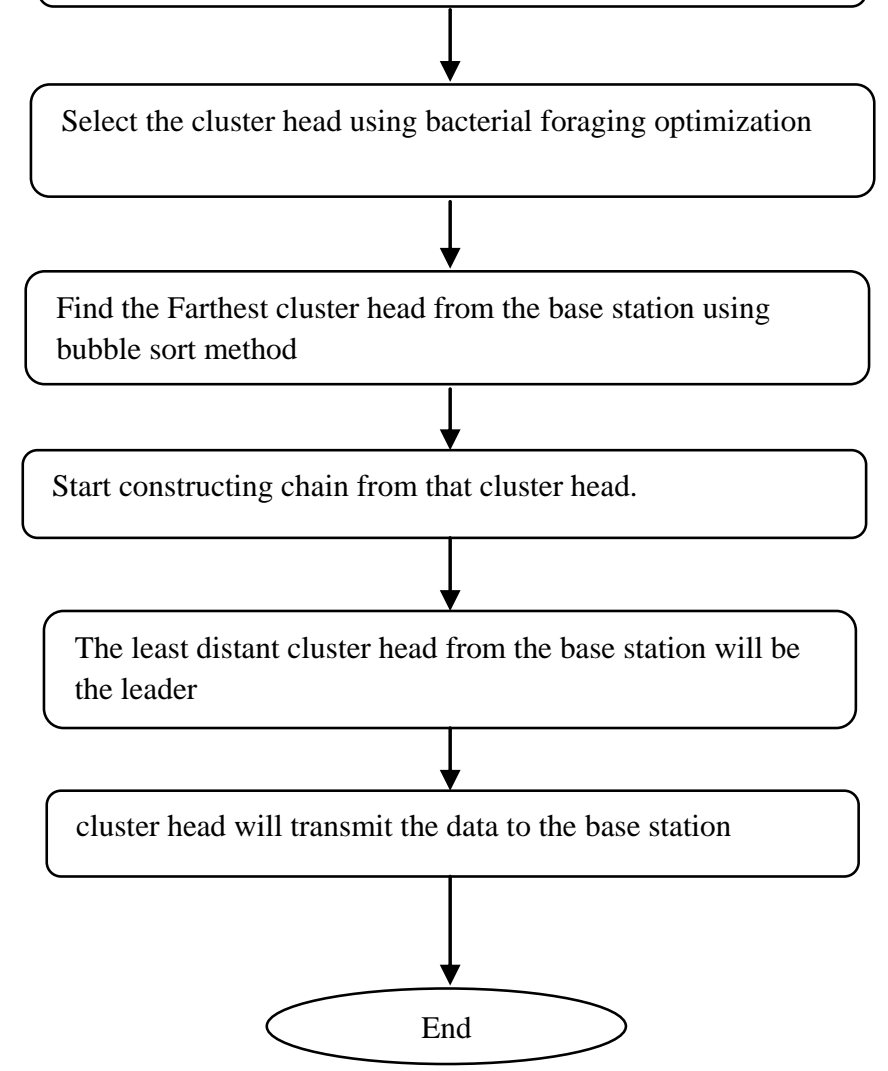

Figure 1.5_Flowchart for working of PEGASIS mechanism

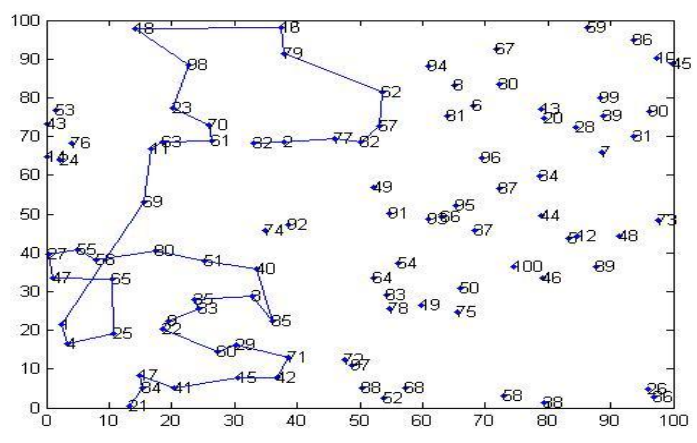

Figure 1.6 Traditional Pegasis chain construction methodology

In above graph 100 nodes are placed randomly. A still fromworking code to show traditional Pegasis chain construction methodology 


\section{SIMULATION RESULTS}

Proposed protocol performs $47 \%$ better on the basis of energy consumption than the existing PEGASIS protocol.

\subsection{Network Lifetime}

Network lifetime can be further classified into two categories:

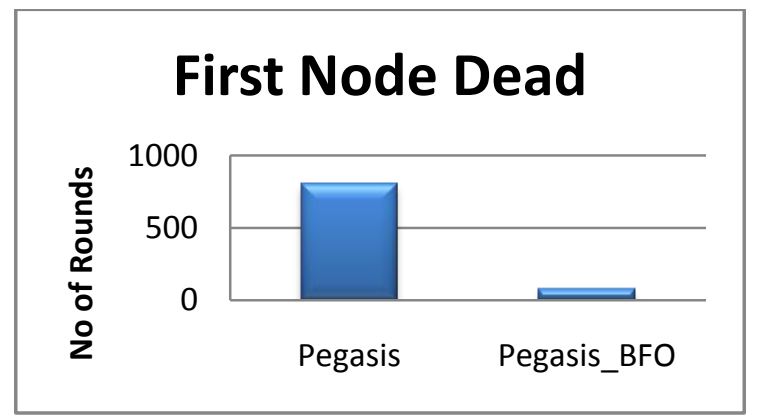

Figure1.7 First node dead

\subsubsection{First node dead-}

Here bar graph represents Pegasis protocol's First node dies at 801 rounds whereas Pegasis with BFO's First node died at 75 rounds only, but as per large networks pegasis with BFO will be more effective that can be shown as Last node alive.

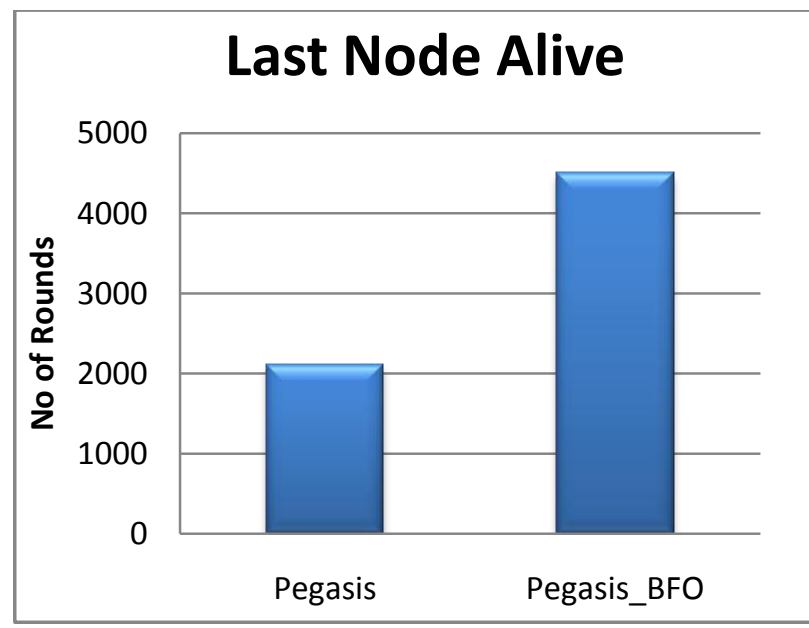

Figure 1.8 Last nodes alive

\subsubsection{Last node alive}

Bar graph represents Pegasis protocol's Last node alive till 2100 rounds whereas Protocol Pegasis with BFO's Last node alive even at 4500 rounds. Which represents that as per large networks Pegasis with BFO is more effective.

\subsection{Energy Consumed}

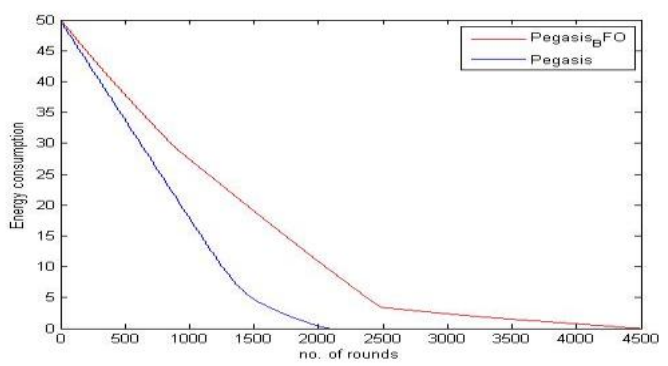

Fig 1.8 Energy consumed by proposed methodology
In this fig, graph represents energy level is degrading constantly for 4500 rounds for Pegasis Protocol and Pegasis with BFO protocol. Here Pegasis Protocol's whole energy is consumed by network in just 2100 rounds whereas Pegasis with BFO performs better than existing Pegasis, as its energy consumption level degraded till 4500 rounds. That means proposed methodology works till 4500 rounds with same energy applied.

\section{ACKNOWLEDGMENTS}

My thanks to the expert Mr. Rajeev (Astt. Professor IT, DAVIET, Jalandhar, Punjab) who have contributed towards development of the paper.

\section{REFERENCES}

[1] Stephanie Lindsey, Raghavendra, Cauligi "PEGASIS: Power-Efficient Gathering in Sensor Information Systems", IEEE, volume-310, pp. 336--1686, 2001

[2] Liu, Tao and Li, Feng "Power-efficient clustering routing protocol based on applications in wireless sensor network", IEEE, pp. 1--6, 2009.

[3] Zhao, Hairong and Zhou, Wuneng and Gao, Yan, "Energy Efficient and Cluster Based Routing Protocol for WSN", Computational Intelligence and Security (CIS) IEEE, Eighth International Conference, pp. 107-$111,2012$.

[4] Gautam, Navin and Pyun, Jae-Young, "Distance aware intelligent clustering protocol for wireless sensor networks" Communications and Networks, IEEE, volume-12, number-2, pp. 122--129, 2010

[5] Elhabyan, Riham S and Yagoub, "Energy efficient clustering protocol for WSN using PSO" Global Information Infrastructure and Networking Symposium (GIIS), IEEE, pages-1--3, 2014

[6] Fan, Xunli and Du, Feifei and Guo, Jun and Zhang, Jie, "Energy independent clustering routing algorithm for wireless sensor network, Fuzzy Systems and Knowledge Discovery (FSKD)" 11th International Conference, IEEE pp.-845-849, 2014.

[7] Sen, Feng and Bing, Qi and Liangrui, Tang, "An improved energy-efficient pegasis-based protocol in wireless sensor networks, Fuzzy Systems and Knowledge Discovery (FSKD)" 2011 Eighth International Conference On, IEEE, volume-4, pages2230--2233, 2011

[8] Nayak, Padmalaya and Anurag, "A Fuzzy Logic based Clustering Algorithm for WSN to extend the Network Lifetime" IEEE, 2015.

[9] Prabhavathi, S and Subramanyam, "Clustering process for maximizing lifetime using probabilistic logic in WSN Computing Communication and Networking Technologies (ICCCNT)" 2014 International Conference on, IEEE, pp. 1-7, 2014.

[10] Aderohunmu, Femi and Deng, Jeremiah D and Purvis, Martin K and others, "A deterministic energy-efficient clustering protocol for wireless sensor networks, Intelligent Sensors, Sensor Networks and Information Processing (ISSNIP)" 2011 Seventh International Conference, IEEE, pp. 341-346, 2011.

[11] Jeet Kamal and Kumar Arun , "Intelligent Cluster Routing Protocol for Wireless Sensor Network based on 
GA", International Journal for Innovative Research in Science and Technology, pp. 4--8, 2015

[12] Nagchoudhury, Pooja and Maheshwari, Saurabh and Choudhary, Kavita, "Optimal Sensor Nodes DeploymentMethod Using Bacteria Foraging Algorithm in Wireless Sensor Networks", Emerging ICT for Bridging the Future-Proceedings of the 49th Annual Convention of the Computer Society of India CSI Volume, Springer, pp. 221-228,2015.

[13] Pitchaimanickam, B and Radhakrishnan, "A hybrid bacteria foraging using Particle Swarm Optimization algorithm for clustering in wireless sensor networks" Science Engineering and Management Research (ICSEMR), IEEE, 2014 International Conference, pp. $1-6,2014$

[14] Bala bantaray, Er Madhusmita and Swain, Er Jyoti Prakash and Dash, Madhumita A "Survey on Coverage Problem in Wireless Ad Hoc and Sensor Networks".
[15] Kavitha, G and Wahidabanu, "FORAGING OPTIMIZATION FOR CLUSTER HEAD SELECTION" Journal of Theoretical \& Applied Information Technology, volume-61, number-3, 2014.

[16] Femi A. Aderohunmu, Jeremiah D. Deng , Martin K. Purvis , "A Deterministic Energy-efficient Clustering Protocol for Wireless Sensor Networks.” IEEE, 2011.

[17] K. M. Passino, "Biomimicry of bacterial foraging for distributed optimization and control," IEEE Control Syst Mag. USA, vol. 22, pp. 52- 67, June 2002.

[18] S. Deng, J. Li, L. Shen, "Mobility-based clustering protocol for wireless sensor networks with mobile nodes"National Mobile Communications Research, IET Wirel. Sens. Syst.,Vol. 1, Iss. 1, pp. 39-47 39, 2011

[19] Z.W. Siew, A. Kiring, H.T. Yew, P. Neelakantan and K.T.K. Teo,"Energy Efficient Clustering Algorithm in Wireless Sensor Networks using Fuzzy Logic Control", IEEE ,2011 